\title{
Analytical Solution of Nonlinear Diffusion Equation Describing Interdiffusion of Gases
}

\author{
A.J. JANAVIČIUSS ${ }^{a, *}$ AND D. JURGAitis ${ }^{b}$ \\ ${ }^{a}$ Faculty of Nature, Šiauliai University, P. Višinskio 19, 76351, Šiauliai, Lithuania \\ ${ }^{b}$ Faculty of Mathematics and Informatics, Šiauliai University, P. Višinskio 19, 76351 Šiauliai, Lithuania
}

(Received February 28, 2008; revised version July 20, 2009; in final form October 12, 2009)

\begin{abstract}
The diffusion is the result of Brownian movement and occurs with a finite velocity. We consider the nonlinear diffusion equation, with diffusion coefficient directly proportional to the impurities concentration. In this case of diffusion from the constant source, the maximum displacements of diffusing particles are proportional to the square root of diffusion time. This result coincides with Brownian movement theory. The obtained analytically solutions were successfully applied for describing the diffusion and superdiffusion experiments' in solids. After theoretical consideration of application of this equation for diffusion in gases, we are investigating here the binary nonlinear diffusion in gases. We obtained the nonlinear interdiffusion equation, for the spherical symmetric case, and presented the approximate analytical solutions.
\end{abstract}

PACS numbers: $66.30 .-\mathrm{h}$

\section{Introduction}

Mass transport, heat conduction, electrical conductivity, crystals growth, and the transport of defects in solids are controlled by diffusion. The system can be transferred to non-equilibrium state where distributions of temperature, pressure and chemical potential depend on coordinates $\boldsymbol{r}$ and are characterized by appropriate gradients $\nabla T(\boldsymbol{r}) \neq 0, \nabla \mu_{i}(\boldsymbol{r}) \neq 0$. The force descended by these gradients generates the diffusion fluxes of mass and heat for every component of multicomponent system [1]:

$$
\frac{\mathrm{d} n_{i}}{\mathrm{~d} t}=\frac{\mathrm{d}}{\mathrm{d} x} \sum_{k} D_{i k} \frac{\mathrm{d}}{\mathrm{d} x} n_{k},
$$

with diffusion coefficients $D_{i k}$, which depends on temperature, concentration of all components and chemical potentials. Those complicated mass transport problems for multicomponent systems can be realized [2] using the Darken equations [3] and numerical methods.

We consider the binary diffusion of gases applying the mathematical methods and analytical solutions, derived for the nonlinear diffusion equation [4-6]:

$$
\frac{\mathrm{d} n}{\mathrm{~d} t}=\frac{\mathrm{d}}{\mathrm{d} x}\left(D \frac{\mathrm{d} n}{\mathrm{~d} x}\right), \quad D(n)=D_{0} n(x, t) .
$$

This equation, with diffusion coefficient directly proportional to impurities concentration, was proposed for calculations of impurities diffusion in silicon. We obtained

\footnotetext{
* corresponding author; e-mail: AYanavy@gmail.com
}

very good coincidence with experiment $[4,5]$. Diffusion equation was introduced [5] using first Fick's law and requiring that diffusion of impurities must emanate with finite velocity and maximum penetration depth into the crystal. This nonlinear diffusion equation in the spherical-symmetric case [6]:

$$
\begin{aligned}
& \frac{\mathrm{d} n}{\mathrm{~d} t}=\frac{D_{0}}{N_{1}}\left[n\left(\frac{\mathrm{d}^{2} n}{\mathrm{~d} r^{2}}+\frac{2}{r} \frac{\mathrm{d} n}{\mathrm{~d} r}\right)+\left(\frac{\mathrm{d} n}{\mathrm{~d} r}\right)^{2}\right], \\
& D_{0}=\frac{1}{2} \sqrt{2} u L^{2} \sigma N_{1}
\end{aligned}
$$

was applied for high-density impurities spread from spherical source into the gases [6] with diffusion coefficient $D_{0}$. Here $N_{1}$ is maximum density of impurities and solvent, $\sigma$ is the collision cross-section, $L$ is the free path of diffusing particles.

\section{Nonlinear equation for binary diffusion in gases}

We assume that the process of penetration of first kind gas into second kind of gas occurs isothermally and is isobaric. We use this simple approach with the aim to get analytical solutions of Darken's equations for binary diffusion $[3,7]$ :

$$
\begin{aligned}
& J_{i}=J_{i \mathrm{~S}}+n_{i} u, \quad J_{i \mathrm{~S}}=-D_{i} \frac{\mathrm{d} n_{i}}{\mathrm{~d} x}, \\
& u=\frac{1}{N}\left(D_{1} \frac{\mathrm{d} n_{1}}{\mathrm{~d} x}+D_{2} \frac{\mathrm{d} n_{2}}{\mathrm{~d} x}\right), \quad i=1,2,
\end{aligned}
$$




$$
\begin{aligned}
& J_{i}=-D_{12} \frac{\mathrm{d} n_{i}}{\mathrm{~d} x}, \quad D_{12}=\frac{n_{2}}{N} D_{1}+\frac{n_{1}}{N} D_{2}, \\
& n_{1}+n_{2}=N, N=\text { const, }
\end{aligned}
$$

transforming it to the nonlinear diffusion equation. $D_{i}$ are intrinsic diffusion coefficients and $D_{12}$ is interdiffusion coefficient for the binary diffusion. The densities of gases are consequently $n_{1}$ and $n_{2}$. In this case there is a problem of evaluation of mean free parts because collisions between the same and different kind of molecules must be included. But also we can use the presented expression (4) for interdiffusion coefficient $D_{12}$. When intrinsic diffusion coefficients are not equal, $D_{1} \neq D_{2}$, the different diffusion fluxes must be compensated by hydrodynamic flux having velocity $u$ and staying constant pressure in the whole volume [7].

Here, from condition $n_{1}+n_{2}=N=$ const we can obtain equation

$$
\frac{\mathrm{d} n_{1}}{\mathrm{~d} x}=-\frac{\mathrm{d} n_{2}}{\mathrm{~d} x} .
$$

We expressed full flux of first kind molecules using (4) and (5)

$$
J_{1}=-D_{12} \frac{\mathrm{d} n_{1}}{\mathrm{~d} x}=-\left[D_{1}+\frac{n_{1}}{N}\left(D_{2}-D_{1}\right)\right] \frac{\mathrm{d} n_{1}}{\mathrm{~d} x},
$$

and connection

$$
\frac{n_{1}}{N}+\frac{n_{2}}{N}=1
$$

Here we introduced, instead $D_{12}$ (interdiffusion coefficient for binary diffusion), the linear part $D_{1}$ and nonlinear part $\left(D_{2}-D_{1}\right) \frac{n_{1}}{N}$ of the coefficient. Using nonlinear diffusion theory for impurities diffusion in gases [6] we can transform linear diffusion equation into nonlinear $[5,6]$ applying Eq. (3) for self-diffusion of first kind molecules. In this case $[5,6]$ we can substitute in (6) $D_{1} \frac{\mathrm{d} n_{1}}{\mathrm{~d} x}$ by $\frac{D_{1}}{n_{1}(0)} n_{1} \frac{\mathrm{d} n_{1}}{\mathrm{~d} x}$. Here we introduced, instead $D_{1}$ the nonlinear coefficient $D_{1 n}$ for nonlinear diffusion of impurities [6]. In this case we can transform Eq. (6) into

$$
\begin{aligned}
J_{1} & =-D_{12} \frac{\mathrm{d} n_{1}}{\mathrm{~d} x} \\
& =-\left[\frac{D_{1}}{n_{1}(0)}+\left(D_{2}-D_{1}\right) \frac{1}{N}\right] n_{1} \frac{\mathrm{d} n_{1}}{\mathrm{~d} x},
\end{aligned}
$$

where $n_{1}(0)$ is the density of first kind molecules at source.

For nonlinear interdiffusion (6) we have the following simplified flux expression:

$$
\begin{aligned}
& J_{1}=-\left\langle D_{12 n}\right\rangle n_{1} \frac{\mathrm{d} n_{1}}{\mathrm{~d} x}, \\
& \left\langle D_{12 n}\right\rangle=\frac{D_{1}}{n_{1}(0)}+\left(D_{2}-D_{1}\right) \frac{1}{N}, \\
& n_{1}(0)+n_{2}(0)=N .
\end{aligned}
$$

When $D_{2}=D_{1}$, the equation transforms into nonlinear equation for impurities diffusion $[5,6]$ in solids or gases.
In the case of three dimensions, when frequency $\nu(x, y, z, t)$ of jumps or collisions of diffusing particles, with the overage free path $L$, depends upon the density $n(x, y, z, t)$ of impurities, the following differential equation:

$$
\frac{\partial n}{\partial t}=L^{2} \nu \Delta n+2 L^{2}(\operatorname{grad} \nu)(\operatorname{grad} n)+L^{2} n \Delta \nu
$$

was derived [6].

In binary diffusion case, only the collisions between the impurity atoms or molecules and molecules of solvent gas are important [6]. Using frequencies expression [6] $\nu_{\mathrm{f}}$ of collisions of diffusing particles on the diffusion front where density of diffusing impurities approximately decreases twice in radial direction, we obtain

$$
\left\langle D_{12 n}\right\rangle=\frac{2 L^{2} \nu_{\mathrm{f}}}{N}, \quad \nu_{\mathrm{f}}=\frac{1}{2} \sigma n(x, y, z, t) u \sqrt{2}
$$

and we can transform Eq. (10) to nonlinear equation for impurities diffusion in gases

$$
\frac{\partial n_{1}}{\partial t}=\left\langle D_{12 n}\right\rangle\left[n_{1} \Delta n_{1}+\left(\operatorname{grad} n_{1}\right)^{2}\right] .
$$

Changing the nonlinear diffusion coefficient $\left\langle D_{12 n}\right\rangle$ in the last equation by interdiffusion coefficient (9), we can transform this equation into nonlinear equation for binary diffusion of gases. Now we can modify the nonlinear diffusion Eq. (12), with nonlinear binary diffusion coefficient, for the three-dimensional spherical case in the following manner:

$$
\begin{aligned}
& \frac{\partial n_{1}}{\partial t}=D_{n}\left[n_{1}\left(\frac{\mathrm{d}^{2} n_{1}}{\mathrm{~d} r^{2}}+\frac{2}{r} \frac{\mathrm{d} n_{1}}{\mathrm{~d} r}\right)+\left(\frac{\mathrm{d} n_{1}}{\mathrm{~d} r}\right)^{2}\right], \\
& D_{n}=\frac{D_{1}}{n_{1}(0)}+\left(D_{2}-D_{1}\right) \frac{1}{N} .
\end{aligned}
$$

\section{The approximate analytical solution of the nonlinear interdiffusion equation}

Introducing new function $f$, divided on the density $n_{1}(0)$ of first gases on the spherical surface of source

$$
f(r, t)=\frac{n_{1}(r, t)}{n_{1}(0)}
$$

and similarity variable

$$
\xi=\frac{r-R}{\sqrt{D_{\mathrm{S}} t}}, \quad D_{\mathrm{S}}=D_{n} n_{1}(0), \quad r \geq R,
$$

in Eq. (13) we obtain the following nonlinear radial diffusion equation:

$$
\frac{\mathrm{d}}{\mathrm{d} \xi}\left(f \frac{\mathrm{d} f}{\mathrm{~d} \xi}\right)+\frac{2}{\xi} f \frac{\mathrm{d} f}{\mathrm{~d} \xi}+\frac{1}{2} \xi \frac{\mathrm{d} f}{\mathrm{~d} \xi}=0 .
$$

Expressing approximate solution of this nonlinear equation of diffusion similarly like in [8]:

$$
f\left(\xi_{1}\right)=a_{1} \xi_{1}+a_{2} \xi_{1}^{2}, \quad \xi_{1}=\xi-\xi_{0}, \quad 0 \leq \xi \leq \xi_{0},
$$

we can transform Eq. (16) into the following form:

$$
\begin{aligned}
& \left(\xi_{1}+\xi_{0}\right) \frac{\mathrm{d}}{\mathrm{d} \xi_{1}}\left(f \frac{\mathrm{d} f}{\mathrm{~d} \xi_{1}}\right)+2 f \frac{\mathrm{d} f}{\mathrm{~d} \xi_{1}} \\
& +\left(\frac{1}{2} \xi_{1}^{2}+\xi_{0} \xi_{1}+\frac{1}{2} \xi_{0}^{2}\right) \frac{\mathrm{d} f}{\mathrm{~d} \xi_{1}}=0 .
\end{aligned}
$$


The presented approximate solution (17) of nonlinear interdiffusion Eq. (18) satisfies the following boundary condition, which defines the impurities concentration at source:

$$
n_{1}(r=R, t \geq 0)=n_{1}(0), \quad f\left(-\xi_{0}\right)=1
$$

and initial condition

$$
\begin{aligned}
& n_{1}\left(r>r_{0}, t=0\right)=0, \quad f(0)=0, \\
& \xi_{1}=0, \quad \xi=\xi_{0}
\end{aligned}
$$

for the case $n_{1}+n_{2}=N$.

Here we used maximum penetration distance $\xi_{0}$ (in radial variable $\left.r_{0}=\xi_{0} \sqrt{D_{\mathrm{S}} t}+R\right)$ of gases, spreading from spherical source, where its concentration becomes equal to zero $[8,9]$.

Substituting function (17) into Eq. (18) and requiring the satisfying of boundary condition (19), we obtained system of nonlinear equations

$$
\begin{aligned}
& a_{1} \xi_{0}+2 a_{1}+\frac{1}{2} \xi_{0}^{2}=0, \\
& a_{1}^{2}+\left(\xi_{0}+2\right)\left(6 a_{1} a_{2}+6 a_{2}^{2}\right)+a_{1} \xi_{0}+\xi_{0}^{2} a_{2}=0, \\
& -a_{1} \xi_{0}+a_{2} \xi_{0}^{2}=1 .
\end{aligned}
$$

Solving this system of equations, we calculated the coefficients

$$
a_{1}=-0.304, \quad a_{2}=0.266, \quad \xi_{0}=1.449,
$$

for sufficiently exact analytical solution of (18). After substitution of the obtained constants into (17) we obtain $f\left(-\xi_{0}\right)=0.999 \approx 1$ and boundary condition (19) is satisfied practically exactly. Using obtained coefficients and Eqs. (20), (19) we can express radial density of diffusing impurities in gases

$$
\begin{aligned}
& n_{1}(r, t) \\
& \quad=n_{1}(0)\left[-0.304(\xi-1.449)+0.266(\xi-1.449)^{2}\right], \\
& \xi=\frac{r-R}{\sqrt{D_{\mathrm{S}} t}}, \\
& D_{\mathrm{S}}=D_{n} n_{1}(0), \quad D_{n}=\frac{D_{1}}{n_{1}(0)}+\left(D_{2}-D_{1}\right) \frac{1}{N}, \\
& 0 \leq \xi \leq 1.449
\end{aligned}
$$

for the spherical source with radii $R$. Here a density of impurities at source $n_{1}(R, t)=n_{1}(0)$ does not depend on time. It is important to underline that diffusion coefficient

$$
D_{\mathrm{S}}=D_{1}+\left(D_{2}-D_{1}\right) \frac{n_{1}(0)}{N}
$$

coincides with coefficient $D_{12}(0)$ for binary diffusion (4) at the infinity spherical source.

We can compare nonlinear binary solution (25) with the analytical solution of the linear diffusion equation [9] for interdiffusion case

$$
\frac{\partial n_{1}}{\partial t}=D_{12}\left(\frac{\mathrm{d}^{2} n_{1}}{\mathrm{~d} r^{2}}+\frac{2}{r} \frac{\mathrm{d} n_{1}}{\mathrm{~d} r}\right)
$$

with the boundary condition for infinity source

$$
n_{1}(r=R, t \geq 0)=N
$$

and initial condition

$$
n_{1}(r>R, t=0)=0
$$

for the case $n_{1}+n_{2}=N$.

This solution can be expressed [9] as follows:

$$
n_{1}(r, t)=N \frac{R}{r} \operatorname{erfc}\left(\frac{r-R}{2 \sqrt{D_{12} t}}\right), \quad r \geq R .
$$

Presented boundary and initial conditions for the solution (30) of linear Eq. (27) coincide with conditions (19) and (20) for solution (25) of nonlinear Eq. (13) when $n_{1}(0)=N$ and $n_{1}+n_{2}=N$. Solution (25) of the nonlinear Eq. (13) defines the maximum penetration $r_{0}=R+\xi_{0} \sqrt{D_{\mathrm{S}} t}\left(\xi_{0}=1.449\right)$ of first kind gas in the surrounding second gas by a finite time of diffusion.

\section{Results and conclusions}

We compared the obtained solution (25) of nonlinear diffusion Eq. (13) with solution (30) of linear radial diffusion Eq. (27) for diffusion of CO in air with interdiffusion coefficient [10] $D_{12}=0.182 \mathrm{~cm}^{2} / \mathrm{s}$. The diffusion coefficient $D_{\mathrm{S}}$ of nonlinear equation is expressed in Eq. (26) by self-diffusion coefficients [10] for CO: $D_{1}=0.175 \mathrm{~cm}^{2} / \mathrm{s}$ and air $D_{2}=0.17 \mathrm{~cm}^{2} / \mathrm{s}$. All diffusion coefficients are presented at normal pressure. The radius of the diffusion source is assumed to be equal to $R=1 \mathrm{~cm}$. The dependence of relative radial concentration $n_{1} / N$ of diffusing $\mathrm{CO}$ gases in air on radial variable are presented in Figs. 1 and 2 in nonlinear and linear cases for diffusion time $t_{1}=10 \mathrm{~s}$ and $t_{2}=100 \mathrm{~s}$, consequently.

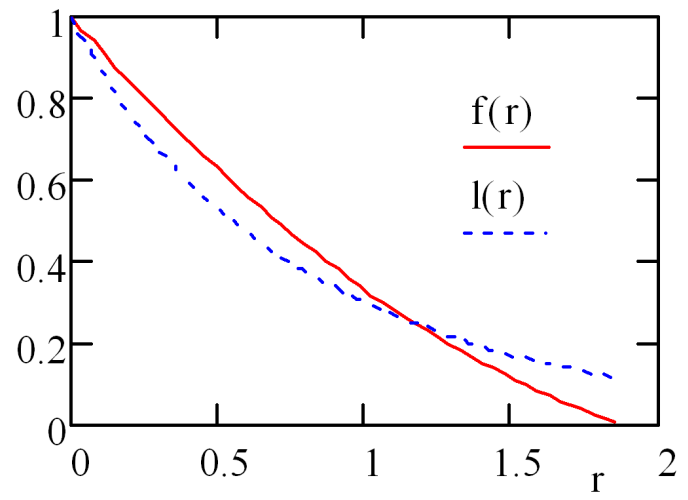

Fig. 1. Dependence of relative radial concentrations $n_{1} / N$ of first kind gas on distance $r \mathrm{~cm}$ from source, expressed by solutions $f(r)$ and $l(r)$ of nonlinear and linear diffusion equations at diffusion time $t=10 \mathrm{~s}$.

Profiles $f$ and $F$ of relative concentrations $n_{1} / N$ of first kind gas, obtained from the analytical solution (25), (14) of nonlinear diffusion Eq. (18), at diffusion time $t_{1}=10 \mathrm{~s}$, $t_{2}=100 \mathrm{~s}$ are presented in Figs. 1 and 2 . The penetration depths of impurities and profiles coincide with the results obtained numerically [6] for the same diffusion parameters. Profile $L=n_{1} / N$ of linear diffusion Eq. (30), 


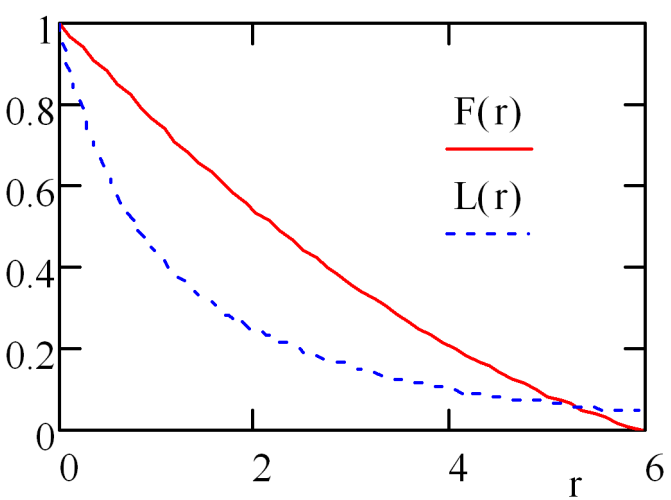

Fig. 2. Dependence of relative radial concentrations $n_{1} / N$ of first kind gas on distance $r \mathrm{~cm}$ from source, expressed by solutions $F(r)$ and $L(r)$ of nonlinear and linear diffusion equations at diffusion time $t=100 \mathrm{~s}$.

except short time diffusion profile $l$ of relative concentrations presented in Fig. 1, has significantly larger curvature than $F$. We observe the largest differences between the nonlinear solutions $F$ and $L$ for long time of diffusion and great distances. The solutions (25) of nonlinear Eq. (13) are exactly equal to zero beyond a certain distance $1.449 \sqrt{D_{\mathrm{S}} t}$ from diffusion source. The solution (30) of the linear Eq. (27) only asymptotically tends to zero.

In the paper we obtained that the Darken equations for binary diffusion can be transformed to the nonlinear interdiffusion equations for gases. Sufficiently exact analytical solutions (25) presented in Figs. 1 and 2 practically coincides with the same numerical solutions obtained in [6] where experimental interdiffusion coefficients were used.

\section{References}

[1] J.P. Stark, Metall. Mater. Trans. A 11, 1073 (1980).

[2] M. Danielewski, M. Pietrzyk, B. Wierzba, Solid State Phenom. 129, 11 (2007).

[3] L.S. Darken, Trans. AIME 174, 184 (1948).

[4] A.J. Janavičius, V. Stukaitè, D.J. Zanevičius, Electron Technique, Ser. 2, Semicond. Dev. 160, 27 (1983) (in Russian).

[5] A.J. Janavičius, Phys. Lett. A 224, 159 (1997).

[6] A.J. Janavičius, A. Poškus, Acta Phys. Pol. A 107, 519 (2005).

[7] Y. Oishi, M. Sugisaki, Y. Kamei, Y. Shono, Bull. Chem. Soc. Japan 45, 2984 (1972).

[8] A.J. Janavičius, P. Norgèla, D. Jurgaitis, Math. Modell. Anal. 6, 77 (2001).

[9] M.E. Gliksman, Diffusion in Solids, Wiley, New York 2000, p. 472.

[10] Laboratory Guide of Physics, Ed. L.L. Goldin, Nauka, Moscow 1983, p. 704 (in Russian). 